\title{
Blocking Reduction of Span Restoration Requests in GMPLS Controlled WDM Optical Networks
}

Buron, Jakob Due; Ruepp, Sarah Renée; Andriolli, N.

Published in:

European Conference on Optical Communications, 2006. ECOC 2006.

Link to article, DOI:

10.1109/ECOC.2006.4801311

Publication date:

2006

Document Version

Publisher's PDF, also known as Version of record

Link back to DTU Orbit

Citation (APA):

Buron, J. D., Ruepp, S. R., \& Andriolli, N. (2006). Blocking Reduction of Span Restoration Requests in GMPLS Controlled WDM Optical Networks. In European Conference on Optical Communications, 2006. ECOC 2006. (pp. 1-2). IEEE. https://doi.org/10.1109/ECOC.2006.4801311

\section{General rights}

Copyright and moral rights for the publications made accessible in the public portal are retained by the authors and/or other copyright owners and it is a condition of accessing publications that users recognise and abide by the legal requirements associated with these rights.

- Users may download and print one copy of any publication from the public portal for the purpose of private study or research.

- You may not further distribute the material or use it for any profit-making activity or commercial gain

- You may freely distribute the URL identifying the publication in the public portal 


\title{
Blocking Reduction of Span Restoration Requests in GMPLS Controlled WDM Optical Networks
}

\author{
Jakob Buron (1), Sarah Ruepp (1), Nicola Andriolli (2)
}

1: COM•DTU, Technical University of Denmark, B345V, 2800 Kgs. Lyngby, Denmark, fjbu, sr\}@com.dtu.dk 2: Scuola Superiore Sant'Anna, Via Moruzzi 1, 56124 Pisa, Italy, nick@sssup.it

\begin{abstract}
The proposed label preference scheme reduces blocking of span restoration requests in GMPLS optical networks with limited wavelength conversion. By minimizing resource contention and conversion usage, it increases recovery percentage and reduces control plane load.
\end{abstract}

\section{Introduction}

Span restoration is a network resiliency technique that provides fast recovery of span failures by setting up a local bypass connection between the failureadjacent nodes [1]. We propose a scheme for blocking reduction of span restoration requests that fits well into the Generalized Multi-Protocol Label Switching (GMPLS) [2] protocol suite, which is a strong candidate for controlling Wavelength Division Multiplexed (WDM) optical networks. For each connection provisioning or recovery in WDM networks, a route and a wavelength need to be found. This is usually a decoupled process, where the connection routing is taken care of by the OSPF-TE protocol, while the RSVP-TE protocol manages resource reservation. Each connection occupies one wavelength and is assigned a label. Wavelengthconverters (WC) remove the requirement for wavelength continuity of the end-to-end path. But WCs are expensive, so only a limited number is provided at each node. If all WCs are occupied, a wavelengthcontinuous path needs to be found, which greatly reduces the probability of successful restoration.

\section{Blocking Reduction}

In the event of a span failure, the recovery is simultaneously initiated for all affected connections. For each one, the upstream failure-adjacent node finds a shortest-path restoration route and then sends a PATH message towards the downstream failureadjacent node, thereby collecting information on available labels along the envisaged route. Once the downstream failure-adjacent node is reached, it chooses a label and sends a RESV message towards the upstream failure-adjacent node. Since no resources are reserved with the PATH message, several recovery requests may want to use the same label, leading to resource contention that can be solved through wavelength conversion. The successful connection setup and the occurrence of forward and backward blocking due to WC unavailability are illustrated in Figure 1. If a span is completely occupied in the timeframe between route calculation and arrival of a PATH or RESV message, blocking occurs even if WCs are available.

This work has been partially supported by E-Photon/ONe+
If forward or backward blocking occurs, the recovery is re-attempted over a different route after a back-off period. Re-routing is performed with information from the GMPLS experimental crankback feature [3].
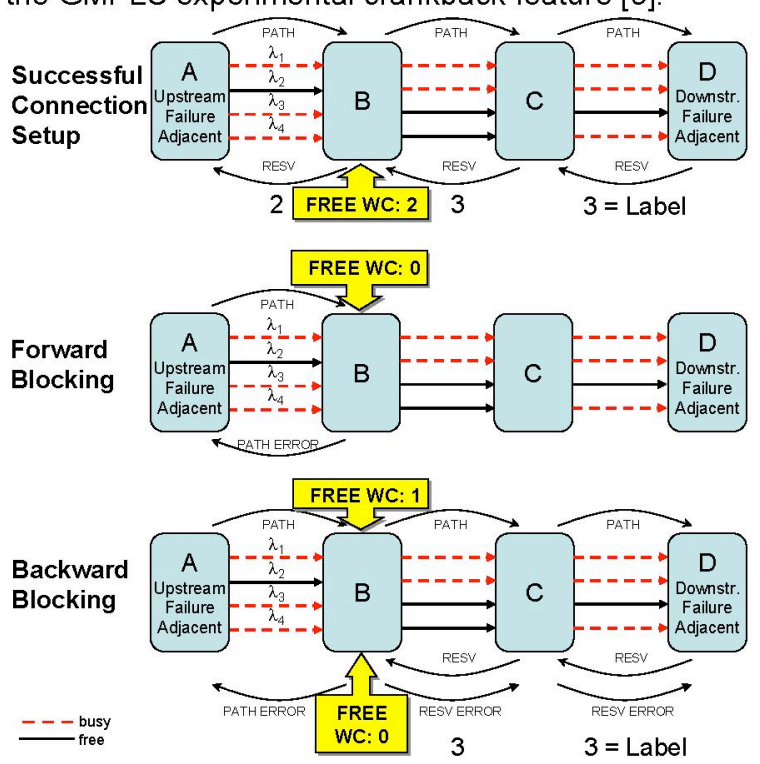

Figure 1: Successful connection setup compared to forward and backward blocking

To minimize the blocking probability and thereby the number of signalling messages and back-offs, the wavelength assignment of the recovery routes should be administrated in a WC-saving manner. To obtain this we use a signalling protocol extension called Suggested Vector (SV) first proposed in [4]. The SV is used in combination with the label set, which is a standard protocol extension allowing an upstream node to control the label selection of a downstream node. By means of the SV, a label preference level is recorded at each node, enabling the downstream node to choose the most preferable label. In the case of equal SV values, ties are broken first-fit.

In the SV scheme, all labels are assigned identical initial SV values, so the downstream failure-adjacent node has a large selection of equally preferable labels to choose from. In a span restoration scenario this behaviour has two drawbacks: the probability of collision with simultaneous recovery attempts increases; moreover wavelength converters may be required at failure-adjacent nodes, if a label different 
from the one used on the failed span (pre-failure label) is chosen. To solve both issues, we propose a modification of the SV scheme, called blocking reduction (BR), where the pre-failure label is the preferred label, with a SV value of 0 . The pre-failure labels of other affected connections are penalized by giving them a SV value of 2 , reducing their chance of being chosen. Unused labels are assigned a SV value of 1 . At each $W C$ en-route, the $S V$ value is increased by 1 . The principle is illustrated in Figure 2.

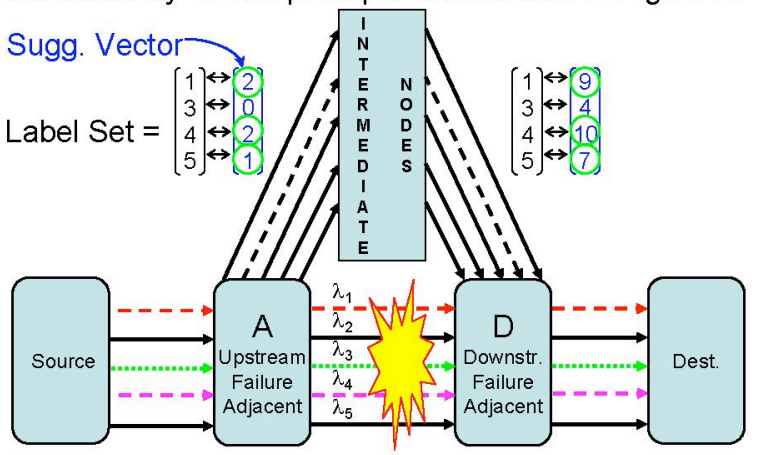

$\equiv \equiv$ Eoccupied by other connections

Chosen label $=3$

Figure 2: SV modified for blocking reduction

\section{Simulation Study and Results}

We investigate the BR scheme's performance in terms of used WC and back-offs for recovered connections, and recovery percentage in comparison to the No Preference (NP) scheme, where a label is chosen amongst free labels on the last hop only, and the aforementioned SV scheme. The schemes are simulated in OPNET Modeler [5] and evaluated on the COST 266 Pan-European triangular topology network [6]. The network consists of 28 nodes and 61 spans, resulting in a nodal degree of 4.36. Each span has a capacity of 10 wavelengths, and each node has 5 WC available (except in Figure 5). The network is populated with unidirectional connections up to a desired average span load using the SV scheme. In this study, 0.6 is the highest load leaving enough capacity for restoration. After reaching the desired load, the spans are consecutively failed. The restoration paths are found by using the Dijkstra shortest path algorithm. If the restoration request experiences blocking, the causing span will be removed and the connection setup will be reattempted over the remaining shortest path after a back-off period of 1 second.

Figure 3 shows that with the BR scheme, the number of used WC decreases compared to the NP and SV schemes, while the connection recovery percentage increases. Figure 4 illustrates that the number of back-offs due to forward and backward blocking is reduced compared to the SV scheme for loads up to 0.5 . At 0.6 load, the higher recovery percentage of $B R$ causes a higher back-off count since only back-offs of recovered connections are included. The reduction of WC usage scales well with the number of WC per node as shown in Figure 5. In all cases, significantly better recovery percentages are achieved.

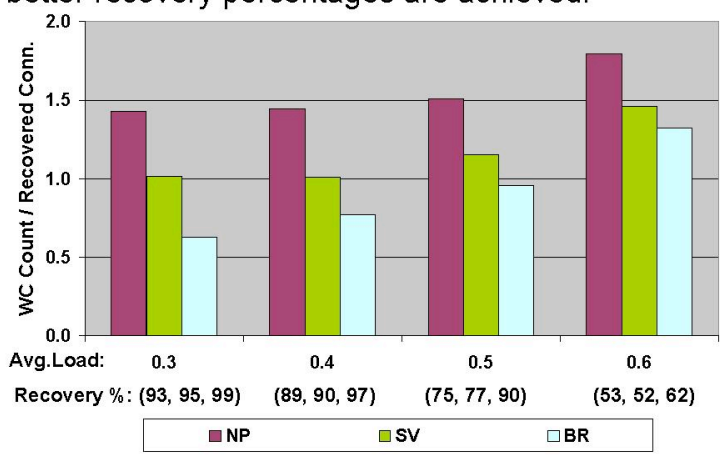

Figure 3: Used WC pr. recovered connection vs. load. Each node has 5 WC.

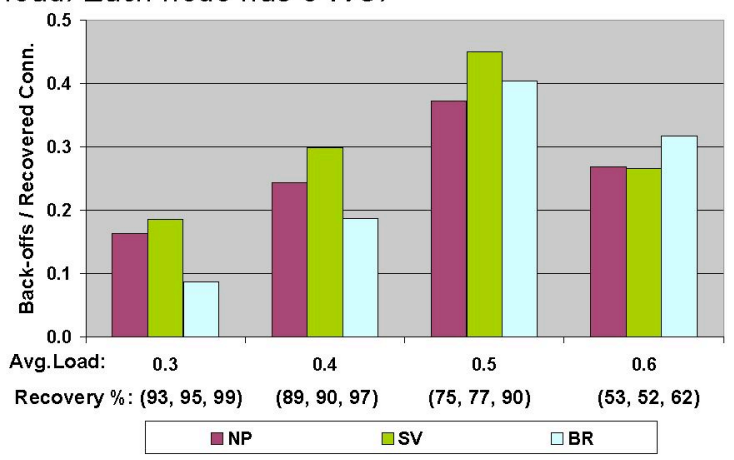

Figure 4: Backoffs (due to for-/backward blocking) pr. recovered connection vs. load. 5 WC per node.

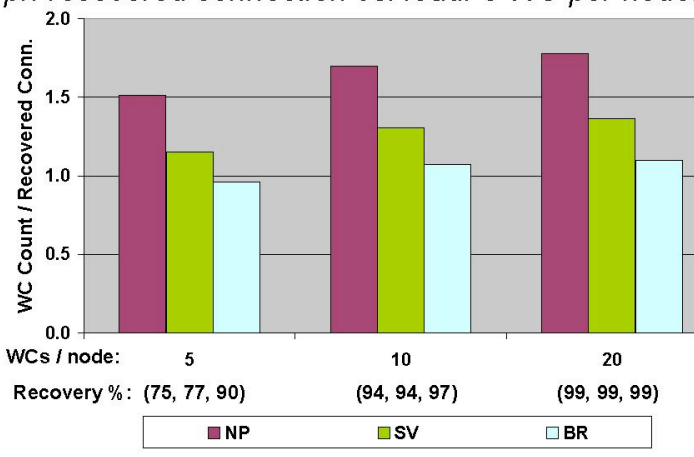

Figure 5: Used WC pr. recovered connection vs. number of WC per node. Load is 0.5 .

\section{Conclusion}

We show that the presented BR scheme for blocking reduction in span restoration is superior to the NP and the SV schemes in terms of used WC, back-offs, and recovery percentage. Fewer blockings and thereby error messages reduce the load on the control plane and increase the restoration speed, since less connections experience back-off and additional route computation.

\section{References}

1 Ramamurthy et al. JLT, Vol. 21, no. 4, April 2003

2 Mannie. RFC 3945, October 2004

3 Farrel et al. draft-ietf-ccamp-crankback, May 2005

4 Andriolli et al. HPSR, Poznan, Poland, 2006.

5 OPNET Modeler, www.opnet.com

6 Inkret et al. Extd. Final Report of COST 266, 2003 